Estudios Románicos, Volumen 28, 2019, pp. 151-164

ISSN: 0210-491

eISSN: 1989-614X

DOI: https://doi.org/10.6018/ER/377011

\title{
LA BIBLIOTECA DEL MONASTERIO DE EL ESCORIAL SEGÚN ALGUNOS VIAJEROS FRANCESES DEL SIGLO XVIII
}

(The Library of the Monastery of El Escorial according to some Eighteenth-Century French Travellers)

\author{
Irene Aguilá-Solana* \\ Universidad de Zaragoza
}

\begin{abstract}
This study is based on a corpus of texts by Vayrac, Saint-Simon, Silhouette, Beaumarchais, Peyron, Bourgoing, and Marcillac, which were published after their visit to Spain, and it analyses how these authors view and describe the Royal Laurentian Library of the monastery of San Lorenzo de El Escorial. These Age of Enlightenment authors tackle a wide range of aspects, such as the layout and decoration of the space, the features of the collection, or the attitude of the monks in charge of the works. While the travellers' level of detail and rigour with regard to the library of El Escorial may vary, their accounts are nevertheless equally interesting since they reveal the different perspectives that they wish to convey to their contemporaries.
\end{abstract}

Keywords: Travel Literature; Enlightenment; Madrid; Diplomacy; Books; Monks.

Resumen: A partir de un corpus formado por los textos de Vayrac, Saint-Simon, Silhouette, Beaumarchais, Peyron, Bourgoing y Marcillac, publicados tras su estancia en España, este estudio analiza las descripciones y opiniones de dichos autores sobre la biblioteca de El Escorial. Los mencionados escritores franceses del Siglo de las Luces abordan numerosas facetas tales como la distribución y decoración del espacio, las características de los fondos allí conservados, el talante de los monjes al cuidado de la Laurentina, etc. Aunque no todos los viajeros son igual de detallistas y rigurosos a la hora de referirse a la biblioteca escurialense, sus testimonios son parejos en interés porque revelan las distintas perspectivas que desean transmitir a sus contemporáneos.

Palabras clave: Literatura de viajes; Ilustración; Madrid; Diplomacia; Libros; Monjes.

\footnotetext{
* Dirección para correspondencia: Irene Aguilá-Solana. Departamento de Filología Francesa. Facultad de Filosofía y Letras. Universidad de Zaragoza. Campus San Francisco. 50009 Zaragoza (iaguila@unizar.es).
} 
Los viajeros franceses que vienen a España durante el siglo XVIII se interesan por numerosos temas, ya por un afán de comparación respecto a su país de origen, ya debido a la curiosidad que en ellos despiertan la historia, las costumbres y la idiosincrasia españolas. Entre las cuestiones que atañen a la cultura, los visitantes galos reparan en las bibliotecas tanto de procedencia privada como de la Iglesia y públicas. En este trabajo nos ocuparemos de las descripciones y opiniones de estos viajeros en lo que concierne a la librería regia, creada por Felipe II en el monasterio de El Escorial ${ }^{1}$. No en vano, la Escurialense, también llamada Laurentina ${ }^{2}$, es el resultado del afán humanista del monarca por fundar una biblioteca tomando como modelo la Vaticana (1450), en Roma, la Marciana o de San Marcos (1560), en Venecia, la Laurenciana o Medicea (1571), en Florencia, o la Ambrosiana (1607), en Milán. El orden seguido en la presentación de los siete autores que forman nuestro corpus respeta la cronología de su paso por Madrid, con el objetivo de comprobar si hay similitudes entre sus juicios y evolución en la naturaleza de sus contenidos. Por esta razón, las palabras de Vayrac, Saint-Simon, Silhouette, Beaumarchais, Peyron, Bourgoing y Marcillac, insoslayables a pesar de la prodigalidad de algunas citas, serán el hilo conductor de este análisis.

No existe datación concluyente sobre la vida y la estancia en España de Jean de Vayrac $^{3}$ donde vino enviado por Luis XIV para que, gracias a su dominio del español, sirviera a su nieto Felipe $\mathrm{V}$ de intérprete y de asesor cuando este llegó al trono. En État présent de l'Espagne (1718), Vayrac dedica una extensa descripción a la biblioteca de El Escorial, que considera una obra maestra engarzada en un microcosmos a imagen de una ciudad $^{4}$. Merece la pena detenerse en la visión del que podríamos calificar como el primer acercamiento de un viajero francés del siglo XVIII a esta insigne librería. El abate la sitúa en el conjunto del edificio ("Au dessus du vestibule est la Bibliotheque" 1718: 519) , alaba la belleza de las estanterías ("Les tablettes sont faites de plusieurs sortes de bois rares apportez des Indes, dont la diversité des couleurs fait un très-bel effet ${ }^{6 "}$ "1718: 536), así como del pavimento ("Le planché est pavé de marbre \& de fayance fine, peinte en bleu, qui fait un beau parterre" 1718: 536). Las ventanas de la sala, por su número y factura, llaman también su atención: "Vingt grandes fenêtres lui donnent tout le jour dont on a besoin pour en voir toutes les beautez: les vitres en sont de crystal, $\&$ se ferment avec de petites targettes d'argent doré" (1718: 536). El visitante es meticuloso al referirse a los fondos (su caudal, su ordenación, sus características) así como a las peculiaridades del préstamo:

1 Para conocer la opinión de Labat, Silhouette, Peyron y Bourgoing sobre otras bibliotecas españolas, ver Aguilá (2017). Blasco (1991), Bonet (1996) y Hernández (1996) reúnen declaraciones de extranjeros en torno a El Escorial sin centrarse en su librería.

2 No hay que olvidar que Felipe II mandó construir el monasterio en reconocimiento por la victoria de San Quintín, que tuvo lugar el día de San Lorenzo de 1557.

3 Basándose en los estudios de Michaud, Feller y Morel-Fatio, Jiménez sitúa el nacimiento del abate en 1664 y su muerte en 1734 o 1735. La estancia en España de Vayrac abarcaría veinte o veinticinco años (2006: 3637). A pesar de que Jiménez afirma (2006: 86) que este autor no figura en el catálogo de García-Romeral, sí que hay una entrada para dicho viajero (García-Romeral 2000: 210).

4 Para describir este palacio-monasterio, sigue casi literalmente Les délices de l'Espagne et du Portugal (1707) de Juan Álvarez de Colmenar (Blasco 1991:171).

5 Se mantiene la ortografía de los textos originales en todas las citas de este trabajo.

$6 \quad$ Ver nota 30. 
Les tremeaux d'entre les fenêtres sont coupez en cabinets sans portes remplis de 8. tablettes, chargées de 8. rangs de Livres tous reliez, \& dorez de la même façon, avec un gril doré au dessus pour Armes ${ }^{7}$. On y compte jusqu'à 130000. volumes ${ }^{8}$, sur toutes sortes de sujets \& sur toutes sortes de Langues [...] Quelques-unes [des] tables sont vuides \& servent à ceux qui souhaitent consulter quelque Livre : car il n'est permis, non pas même aux Religieux, d'en emporter aucun. [...] De la salle où sont les Livres imprimez, on passe par une gallerie dans une autre, où l'on voit 14000. ou 15000. volumes de Manuscrits ${ }^{9}$ [...]. Ils sont tous reliez d'un velours ras $\&$ disposez comme dans des rayons. Les plus estimez sont un Saint Chrysostome en Grec; un Traité de Saint Augustin touchant le Bâteme, écrit de la main de ce Docteur de l'Eglise [...]; un autre écrit de la main de Sainte Therese; un autre écrit sur des feüilles de palmes, \& un autre écrit en lettres d'or, qui contient les 4. Evangiles avec les Préfaces de Saint Jerôme, \& les Canons d'Eusebe ${ }^{10}$, qu'on dit avoir été fait du tems de l'Empereur Conrad. Mais ce qu'il y a de plus remarquable, est une Bibliotheque entiere de Livres Arabes, qu'un Capitaine de vaisseau vola au Roi de Maroc il y a environ 100. ans, \& qu'il vendit au Roi d'Espagne. On tient qu'elle est composée de 7800. volumes ${ }^{11}$ (1718: 536-539).

Asimismo, al describir las curiosidades que encierra, pone en evidencia cómo los cuadros de los muros y la decoración pictórica de su bóveda subrayan la voluntad de unir arte y literatura. Los objetos científicos exhibidos en la sala van acordes con la concepción de espacio cultural integral ideado por Felipe II $^{12}$.

Entre la voûte \& les cabinets, on voit les portraits des quatre premiers Rois d'Espagne de la Maison d'Autriche, \& ceux de plusieurs grands hommes, dont le

$7 \quad$ Saint-Simon, por su parte, explica el origen de esta figura en su relación con El Escorial: "La forme de gril a réglé toute l'ordonnance de ce somptueux édifice, en l'honneur de saint Laurent et de la bataille de SaintQuentin, gagnée la veille [10 août 1557] par Philippe II [...]. Il n'y a portes, serrures, ustensiles de quelque sorte que ce soit, ni pièce de vaisselle qui ne soit marquée d'un gril" (1858: 352).

8 Las cifras son a todas luces exageradas. Según los inventarios antiguos, las obras impresas conservadas en el siglo XVIII en la Laurentina serían del orden de los 45.000 ejemplares (RBME n.d.: "Composición del fondo bibliográfico impreso").

9 Vayrac abulta también esta cantidad; en conjunto habría en aquel tiempo más de ocho mil manuscritos (RBME n.d.: "Manuscritos árabes", "Manuscritos castellanos", "Manuscritos griegos", "Manuscritos hebreos", "Manuscritos latinos").

10 Entre los libros de la segunda remesa que llegaron a El Escorial en 1566, se encuentran "el famoso Códice Aureo, que contiene los Evangelios en letras de oro, el célebre Apocalipsis Figurado de la casa de Saboya, el manuscrito De baptismo parvulorum, de San Agustín, que se creía autógrafo, al igual que unos Evangelios griegos que se atribuían a la pluma de San Juan Crisóstomo" (Andrés 1970: 10).

11 La cifra que señala Vayrac vuelve a ser excesiva porque Felipe III "después de la muerte de Felipe II, siguió engrosando la Biblioteca con diversas adquisiciones, como [...] sobre todo la cuantiosa biblioteca de códices árabes del Sultán de Marruecos Muley Zidán, apresada por los navíos de Luis Fajardo cerca de Agadir en 1612 y depositada en El Escorial en 1614; el número de volúmenes se aproximaba a los 4.000” (Andrés 1970: 14-15).

12 En efecto, el rey había reunido biblioteca, museo y laboratorio, "a saber, colección de libros, retratos de personajes, conjunto de grabados y dibujos, aparatos geográficos, como mapas, esferas, astrolabios, instrumentos matemáticos y científicos, monetarios, reproducciones de la fauna y flora, etc.” (Andrés 1970: 10). 
nom a été rendu célebre par leur science \& par leurs ouvrages ${ }^{13}$. [...] La voûte est embellie de peintures parfaitement belles, qui représentent toutes les Sciences \& les sept Arts libéraux, chacune avec son hierogliphe: \& leur rapport avec les Livres est si régulierement observé, que la peinture de chaque Science est posée justement au dessus des Livres qui en traitent ${ }^{14}$. Le milieu de la Salle est occupé d'espace en espace par dix ou douze grandes tables de jaspe enchâssées les unes dans l'ébene \& d'autres dans l'yvoire [...]. On y remarque entre autres choses une Sphere de bronze, qui représente les divers mouvemens des Corps célestes. [...] On y montre encore diverses raretez fort curieuses, comme une pierre d'aimant du poids de sept livres, qui soûtient 25. livres pesant; [...] Parmi une infinité de belles peintures dont cette salle est ornée, on voit le portrait de Don Jean d'Autriche, avec les deux grands fanaux que ce Prince remporta sur la Capitane des Turcs à la bataille de Lepante ${ }^{15}$ (1718 : 536-539).

De la misma manera que ocurre con el número de volúmenes, las dimensiones que constan en su relato son erróneas. Vayrac dice "C'est une salle longue de 194. pas, large de 32. \& haute de 36" (1718: 536), pero, sabiendo que, en realidad, las medidas son "54 m. de largo, $9 \mathrm{~m}$. de ancho y $10 \mathrm{~m}$. de alto" (RBME n.d.: "El recinto"), se comprueba que incluso el gradus, unidad de longitud que toma como base el paso simple $(0,7405$ $\mathrm{m}$.), excede en mucho las magnitudes propuestas. Por otra parte, el hecho de que Peyron aporte en su texto de 1783 unos valores métricos correctos, conservando idénticas cifras que Vayrac aunque sustituyendo el 'paso' por el 'pie' $(0,2962)$, nos lleva a pensar que, quizás, el abate indicara en latín dicha unidad de medida (pes) y que el impresor lo interpretara como el vocablo francés 'pas'.

Motivos políticos traen asimismo a Saint-Simon (1675-1755) a España. Enviado por el regente Felipe de Orleans, llega a Madrid el 21 de noviembre de 1721 con el fin de llevar a cabo las negociaciones para los esponsales entre la infanta María Ana Victoria (hija de Felipe $\mathrm{V}$ e Isabel de Farnesio) y Luis $\mathrm{XV}^{16}$. El 2 de diciembre se desplaza a El Escorial y permanece allí tres días, que se le antojan breves para poder admirar las inmensas riquezas contenidas en el complejo arquitectónico, pero que, a la par, le parecen largos por culpa de la actitud del religioso que les hace de guía. A pesar de poseer cartas de recomendación del nuncio, del rey y de su ministro para tener libre acceso a las "merveilles de ce superbe et prodigieux monastère", Saint-Simon se queja del trato recibido por parte de los jerónimos que allí residen a los que menosprecia e insulta: "Encore verra-t-on que

13 Benito Arias Montano "ordenó que el contorno de la biblioteca provisional se adornara con retratos de personajes ilustres en las letras" (Andrés 1970: 13).

14 La inspiración literaria de la ornamentación y los motivos pictóricos del gran Salón de la Biblioteca escurialense se deben a Fray José de Sigüenza, primer gran cronista de la Orden Jerónima y del Monasterio de El Escorial "y su realización sobre los techos a los pintores Peregrín de Peregrini y sus discípulos, especialmente Bartolomé Carduchi” (Andrés 1970: 14).

15 Dato contradictorio puesto que, según Andrés, los faroles de metal dorado de la capitana del turco apresados en dicha batalla naval se derritieron en el incendio de 1671 (1970: 16). Si así fue, es poco probable que Vayrac los viera en El Escorial. A propósito de este percance, que destruyó una parte de los fondos, hemos observado que existe un asombroso silencio general en todos los textos de los viajeros estudiados.

16 Compromiso matrimonial roto en 1725. 
je ne laissai pas d'éprouver la rusticité et la superstition de ces grossiers hiéronimites [...] fort oisifs, ignorants, sans aucune austérité" (1858: 351). Las ofensas del visitante recuerdan la polémica suscitada en tiempos de Felipe II, debido a que el poder real benefició a la Orden Jerónima al encomendarle el monasterio. Muy pronto, la Compañía de Jesús, deseosa de ser la depositaria de los fondos de la librería, alzó su voz contra los jerónimos, alegando que una congregación dedicada sobre todo a la oración no era apta para atender el que estaba destinado a ser un núcleo cultural de referencia. Siguiendo esta tónica, Saint-Simon expresa una opinión negativa al respecto: "La bibliothèque ne me satisfit point, et les bibliothécaires encore moins" (1858: 353), aunque sin justificar los motivos de su consideración. Creemos que el aristócrata emite un juicio a la ligera; antes bien, numerosas crónicas concluyen que los religiosos que ocuparon dicho cargo a lo largo de los siglos XVI, XVII y XVIII realizaron una gran tarea ${ }^{17}$.

Étienne de Silhouette (1709-1767), quien estuvo en la Península cumpliendo también labores diplomáticas desde el 31 de agosto de 1729 hasta diciembre de ese mismo año, piensa que uno de los atractivos que encierra esta "belle masse de pierres" (1770: 140) es la biblioteca, por su arquitectura, por los lienzos que allí se exhiben y por sus fondos. No facilita detalles concretos; solo alude al elevado valor de los libros y a la insensibilidad para apreciarlos de los monjes que los custodian. Al igual que SaintSimon, Silhouette se hace eco de la campaña de desprestigio contra los jerónimos a los que califica de lerdos, incultos y aprovechados. Del mismo modo lamenta no haber tenido tiempo ni opción para evaluar personalmente el caudal bibliográfico.

Ils ont une très-belle bibliotheque, non-seulement par rapport à la beauté du vaisseau $\&$ des peintures, mais aussi par rapport au nombre \& au choix des Livres. Les Moines n'en font pas d'usage, ils sont fort ignorans, ils ne connoissent pas les trésors qu'ils possèdent, \& je n'ai eu ni le tems, ni la facilité d'en faire par moi-même une recherche exacte. Le plus grand mérite de ces Moines est d'officier avec dignité \& d'être fort riches ${ }^{18}$ : ils sont deux cens ${ }^{19}, \&$ tous parfaitement bien logés, tandis que le Roi l'est fort mal (1770: IV, 139-140).

Más de tres décadas después, Beaumarchais ${ }^{20}$ es más pródigo en detalles que sus inmediatos predecesores. En su carta escrita desde Madrid al duque de La Vallière,

17 El primer bibliotecario comisionado a cargo de la librería, de las pinturas, del archivo y de las escrituras de la casa fue el P. Juan de San Jerónimo. A su muerte, en 1591, le sucede el P. José de Sigüenza y, después de él, el P. Lucas de Alaejos, ambos competentes y cultos. Este último falleció en 1631. En 1725, fue nombrado bibliotecario mayor el P. Antonio de San José, hombre de extraordinario tesón que trabajó durante veinticinco años en la organización, clasificación y catalogación de los 4.500 códices (Andrés 1970: 11, 14-16).

18 "Se dice que los frailes eran muy ricos, tenían dilatadas posesiones, pingües heredades [...]. Tuvieron muchas posesiones, es verdad, pero también lo es, en pluma del padre Sigüenza, que no se sabe qué mayorazgo haya dado tantas limosnas y repartido entre los pobres como cualquiera de las casas de San Jerónimo" (Las Palmas 1963: 415).

19 "A mediados del siglo XVIII, residían en el Monasterio doce Maestros jubilados y unos setenta monjes teólogos, predicadores de S.M., poetas y escritores" (Las Palmas 1963: 408).

20 Viajó a España en 1764 para vengar a su hermana Marie-Louise-Isabelle (familiarmente, Lisette) de la afrenta de José Clavijo y Fajardo por haberle prometido matrimonio en dos ocasiones y no cumplir su palabra. El escritor residió en Madrid durante un año empeñado en diferentes negociaciones financieras con las que pretendía lucrarse. 
fechada en diciembre de 1764, el dramaturgo cree que los libros y manuscritos que contiene la renombrada biblioteca serían más útiles y apreciados en Francia que en España a causa de las distintas inquietudes culturales. A raíz de esta circunstancia, Beaumarchais critica la organización deficiente que observa en la Laurentina. Tanto es así que incluso "le cellier des moines qui gardent ces livres [lui] a paru mieux tenu, plus visité et plus exactement étiqueté" 21 (1858: 504), lo que abunda en la opinión despreciativa manifestada por Saint-Simon o Silhouette. Otro detalle que deja traslucir el pensamiento de Beaumarchais sobre la formación intelectual de los españoles es su sorpresa al saber que, en el monasterio, están proscritos la mayoría de filósofos franceses contemporáneos, en especial Voltaire ${ }^{22}$, censurado tanto por las obras publicadas cuanto por las de futura aparición.

J'ai visité avec beaucoup de soin la bibliothèque fameuse du palais de San Lorenzo, appelé par corruption l'Escurial. Je crois, monsieur le Duc, avoir entendu dire à M. de Grimaldi qu'il vous avait envoyé le catalogue des livres et manuscrits qui la composent. Comme il y a ici beaucoup plus d'esprit que d'acquit, ces beautés si précieuses pour nos savants ne sont en ce pays que l'objet d'une stérile curiosité. [...] Un de ces religieux fort honnête m'a fait présent d'un gros Pétrarque très-ancien, mais cela n'a pas assez de barbe ${ }^{23}$ pour mériter une place au château de Montrouge ${ }^{24}$. Une des choses qui m'a le plus frappé dans ce très-magnifique couvent, c'est la condamnation des livres de presque tous nos philosophes modernes qui est affichée publiquement auprès du chœur des moines ${ }^{25}$ (1858: 503-504).

A continuación, es Jean-François Peyron (1748-1784) ${ }^{26}$, secretario de embajada en Madrid desde 1777 hasta 1778, quien reconoce la magnificencia de El Escorial ${ }^{27}$, cuyas instalaciones lo asemejan a una gran ciudad. Recordemos que Vayrac ya lo describía, a principios de siglo, como un microcosmos. Entre sus edificios, Peyron menciona dos bibliotecas de cuyos fondos, mobiliario, dimensiones y decoración efectúa una descripción bastante pormenorizada:

21 De nuevo, la perspectiva del visitante es sesgada: "El P. Sigüenza cambió las signaturas ideadas por Arias Montano por otras más prácticas, de tres números: el primero indica el estante, el segundo (en romanos) señala el plúteo y el tercero marca el volumen" (Andrés 1970: 14).

22 Peyron indica también que la Inquisición condenó no solo todas las obras que Voltaire había escrito sino todas aquellas que en el futuro pudiera componer.

$23 \mathrm{Si}$, según la voz barbe de la Encyclopédie, la barba es la primera marca de pubertad, es decir, de alguien que todavía no ha alcanzado plena madurez, la expresión "ne pas avoir assez de barbe" podría significar que Beaumarchais otorga poco valor a ese ejemplar de Petrarca que el monje le regaló.

24 Aunque sigue siendo propietario del castillo de Champs-sur-Marne, La Vallière se traslada hacia 1750 al que había hecho construir en Montrouge. "C'est dans son château à Montrouge qu'il réunit sa collection de livres, la plus belle qu'eût jamais possédé un particulier” (Weiss 1841: 225).

25 Había una sala, encima del Salón de los Frescos, destinada a guardar los libros prohibidos, los manuscritos de lenguas modernas y los duplicados (Andrés 1970: 14).

26 Anteriormente había ocupado ese mismo cargo en Bruselas en 1774.

27 Su relación retoma a menudo informaciones del Viage de España (1772) de Antonio Ponz (Blasco 1991: 172). 
Il y a deux bibliotheques dans l'Escurial; la seconde, dont je parlerai d'abord, parce qu'elle n'est pas ouverte au public, \& qu'elle n'est pas aussi bien décorée que l'autre, renferme plus de quatre mille manuscrits, hébreux, grecs $\&$ arabes, \& environ six mille volumes imprimés ${ }^{28}$. La bibliotheque basse, ou celle qui est ouverte à tous ceux qui veulent s'instruire, est une salle longue de cent quatre-vingt-quatorze pieds, large de trente-deux, \& haute de trente-six; elle est pavée de carreaux de marbre bleu $\&$ blanc $^{29}$. Les tablettes qu'elle renferme, \& dont la base est un socle de jaspe d'environ un pied de haut, sont faites des bois les plus précieux de l'Europe $\&$ des Indes ${ }^{30}$; elles sont ornées de colonnes cannelées d'ordre dorique, qui sont au nombre de soixante $\&$ dix, \& qui ont six pieds de hauteur, sans compter les bases \& les chapitaux; elles servent à diviser les diverses matieres qui sont aussi désignées par de belles peintures à fresque, soit historiques, soit allégoriques. Peregrino Tibaldi ${ }^{31}$ peignit au plafond les arts libéraux en figures si colossales qu'elles rapetissent la salle, \& surtout les sujets peints au-dessus des tablettes; son ouvrages ( ic ) n'en est pas moins très-estimé; la philosophie est désignée par Socrate, Platon, Aristote \& Séneque; la théologie par des Peres de l'église ${ }^{32}$. Cette bibliotheque ne contient guere que douze mille volumes: parmi les manuscrits, ceux dont on parle le plus, sont les quatre Evangélistes écrits en lettres d'or; le traité de Saint Augustin de Baptismo Parvulorum, écrit de sa main; les œuvres originales de Sainte Therese; une lettre de Saint Vincent Ferrier au roi Fernand d'Aragon, \&c. Il y a dans la longueur de la salle cinq tables de marbre \& deux de porphyre, qui portent des monuments assez curieux (1783: II, 114-115).

Mas no todos los libros conservados en el monasterio se hallan en su biblioteca porque, según el viajero, en los anaqueles del antecoro habría doscientos catorce libros destinados a los oficios, de gran grosor y en pergamino. Tanto el número de volúmenes ${ }^{33}$ y su descripción física como la relación de tipos de madera con los que se realizó el mobiliario concuerdan con la realidad. Dice Peyron que estos cantorales fueron minia-

28 Ver notas 8 y 9.

29 Vayrac dice que el pavimento es de mármol y de porcelana azul (1718: 536).

30 "Las maderas empleadas fueron muy ricas y tan especiales como la ácana de color y el naranjo, la caoba macho y hembra y el terebinto de color muy claro, el boj, el ébano y el nogal. Todas ellas fueron procuradas y entregadas por el propio Rey y se conservan en su estado natural, sin aceite ni barniz alguno" (Feduchi 1963: 524).

31 Se trata de Pellegrino Tibaldi (1527-1596), "llamado normalmente durante su estancia en España Pellegrino Pellegrini” (Scholz-Hansel 1992-1993: 390-391). Feduchi se refiere a él como Pelegrin de Pellegrini, el "Tibaldi" (1963: 523).

32 "Este salón tiene una cubierta de bóveda de cañón dividida en siete tramos. En ellos se sigue el procedimiento frecuente de colocar el tema principal en el compartimento central: las siete Artes Liberales en forma de matronas: Gramática, Retórica y Dialéctica -el Trivium- y Aritmética, Música, Geometría y Astrología -el Quatrivium-. Y las escenas subordinadas -los personajes insignes y las historias relacionadas con cada una de las ciencias- en su parte inferior. De hecho, en el friso que va desde la cornisa hasta la parte alta de las estanterías se pintaron 14 escenas: dos relativas a cada arte liberal. Y encima de la cornisa, cada arte liberal va acompañada de cuatro sabios, elegidos entre los más insignes y representativos de cada ciencia. El plan general de la Biblioteca se completa con los dos testeros, con las personificaciones de la Filosofía [...] y la Teología" (RBME n.d.: "Pinturas al fresco").

33 Unos doscientos veinte cantorales (Andrés 1970: 61). 
dos con gusto y fineza por Fray Andrés de León y Fray Julián de la Fuente del Saz ${ }^{34}$, ambos hábiles iluminadores del siglo XVI (1783: II, 103), lo cual también es cierto. Cabe señalar aquí el error desafortunado que comete Peyron o el impresor en el nombre del segundo artesano debido a las acepciones del término utilizado ya que, en el texto francés, pone "Fiente", es decir, 'excremento', en vez de "Fuente". Por otro lado, el diplomático juzga de manera subjetiva las traducciones que lleva a cabo Casiri ${ }^{35}$. Se trata de una serie de manuscritos árabes en torno a las dinastías moras y los reyes cristianos $\mathrm{y}$, aunque las historias de sendos pueblos le resultan igual de confusas, el viajero se posiciona ligeramente del lado de los árabes cuando habla del sometimiento y expulsión a los que estos fueron obligados por los vencedores. Elogia la obra de Casiri porque ilustra el talento de los árabes en todos los ámbitos en los que sobresalieron; no obstante, "quelque exacte qu'elle doive être, on regrette d'y trouver trop de précision, \& d'avoir encore, après l'avoir lue, beaucoup à desirer" (1783: I, 24-25).

Una parte de la estancia de Peyron coincide con la de Jean-François de Bourgoing (1748-1811), vinculado a la embajada de Francia en Madrid desde 1777 hasta 1793, al inicio, como primer secretario y, en los dos últimos años, como ministro plenipotenciario. El barón es todavía más prolijo que Peyron a la hora de pormenorizar su visita a la Escurialense. Mediante una narración extremadamente copiosa, similar a la que reserva a la mayoría de cuestiones tratadas en su relato por otros lugares de España, alterna comentarios sobre el volumen y calidad de los fondos, el exceso de decoración que abigarra el espacio en el que se conservan los libros, las referencias a los cuadros, mesas y objetos preciosos allí ubicados, con menciones a autores y eruditos (Casiri, Arias Montano) relacionados con esta Real Biblioteca:

Au premier repos de cet escalier, on trouve des petits cloitres qui conduisent à la Bibliotheque de l'Escurial, moins remarquable par le nombre de ses livres que par leur choix, \& sur-tout par la quantité de manuscrits Arabes \& Grecs qu'elle contient. Tout les Arts ont concouru à sa décoration; $\&$ si elle a un défaut, c'est peut-être qu'elle est trop ornée. La peinture s'est emparée de tout l'espace que n'occupent pas les livres: son plafond qui est voûté, est surchargé d'arabesques \& de figures la plupart colossales. Tibaldi, le maître de Michel-Ange, y a déployé la vigueur souvent exagérée de son pinceau; ses attitudes forcées ressemblent à des contorsions; ses formes, à force d'être grandes, sont gigantesques \& presque monstrueuses; ce qui nuit à l'effet de l'ensemble, en rapetissant le beau vaisseau de la Bibliotheque, \& en écrasant ses autres décorations. Les tablettes qui contiennent les livres, \& qui sont de bois précieux sculpté avec soin, paroissent mesquines au-dessous des colosses de

34 Sobre la escuela de miniaturistas de los monjes jerónimos de El Escorial, ver Álvarez 1930:11.

35 Miguel Casiri de Gartia o de Gaeta, en árabe Mija’il al-Gaziri (1710-1791) fue doctor en Teología y Académico honorario de la Real Academia de la Historia desde 1748 y censor de la misma en 1761. Trabajó como intérprete del reino y tuvo un cargo en la Biblioteca Real patrocinado por el erudito ilustrado Gregorio Mayáns y Siscar, que era su director. Protegido por Campomanes, que fue además discípulo suyo en el aprendizaje de esta lengua, compuso Bibliotheca Arabico-Hispana Escurialensis (Madrid 1760-70), catálogo que clasifica por temas y describe 1851 manuscritos árabes de la Biblioteca del Monasterio de San Lorenzo de El Escorial. 
Tibaldi. Il regne au-dessus d'elles des peintures à fresque de Barthelemi Carducho, qui perdent aussi à ce rapprochement; elles rappellent des traits de l'Histoire sainte ou profane, qui sont relatifs à la science dont les tablettes inférieures offrent les ouvrages. Ainsi, le Concile de Nicée est représenté au-dessus des livres qui traitent de la Théologie; la mort d'Archimede au siege de Syracuse, indique ceux qui ont rapport aux Mathématiques: le plaidoyer de Cicéron, en faveur de Rabirius, les ouvrages relatifs à l'éloquence du Barreau, \&c. Le milieu de la Bibliotheque est occupé par des globes \& des tables: sur l'une d'elles est une petite statue équestre de Philippe IV; sur l'autre un petit temple d'argent massif, orné de lapis-lazzuli \& de pierres précieuses. Tout à l'entour sont rangés tous les aïeux de la Reine Anne de Neubourg, femme de Charles second, jusqu'à Charlemagne, qui est placé au centre du temple. Dans les intervalles des tablettes, on remarque les portraits de Charles-Quint \& des trois Philippes, ses successeurs sur le Trône d'Espagne ${ }^{36}$. [...] Les manuscrits ne se conservent pas dans la grande Bibliotheque qui s'ouvre à tout venant, mais dans une grande salle toujours fermée, qui est précisément au-dessus, \& où l'on relégue tous les livres proscrits par l'orthodoxie espagnole ${ }^{37}$. Autour de cette salle sont suspendus les portraits d'une grande partie des Espagnols qui se sont distingués dans les Sciences $\&$ dans les Lettres, \& qui sont beaucoup plus nombreux que ne le croit notre légéreté dédaigneuse. [...] Un petit cabinet attenant à cette salle contient plusieurs reliques, une des urnes miraculeuses des nôces de Cana, un vieux manuscrit de la vie de Sainte Thérese, écrite par elle-même, \&c. (1788: 188-193 y 195-196).

Una de las diferencias más importantes respecto al discurso de otros viajeros anteriores radica en la reflexión de Bourgoing acerca de los prejuicios de sus compatriotas en lo tocante a los españoles, en general, y a los monjes, en particular. En su opinión, hay que desconfiar de los modales finos que enmascaran la hipocresía francesa y apreciar la ruda bondad de los jerónimos. Por ello, al hilo de la pintura de Pantoja de la Cruz expuesta en la biblioteca y que representa a Felipe II, Bourgoing alude a la austeridad del monarca y de su reinado para contraponerla a la largueza de estos religiosos a la hora de acoger a los usuarios: "La Bibliotheque de l'Escurial est ouverte soir \& matin

36 "En las columnas de los dos arcos principales de la sala hay colocados cuatro retratos de tamaño natural: el Emperador Carlos V a la edad de 49 años, copiado muy fielmente por Pantoja de la Cruz del original de Tiziano que se conserva en el Museo del Prado; enfrente Felipe II a la edad de 71 años, atribuido al mismo pintor. En el segundo arco, Felipe III a la edad aproximada de 28 años, obra de Juan Pantoja de la Cruz; en el lado opuesto se encontraba un soberbio retrato de Felipe IV, pintado por Diego Velázquez, que desapareció durante la Guerra de la Independencia; José Bonaparte se lo regaló al general francés Desolle y en la actualidad se puede admirar en la National Gallery de Londres" (RBME n.d.: "Pinturas al óleo").

37 Bourgoing habla de una gran sala, además de la principal, cuando, en realidad, "[e]l conjunto de la Biblioteca disponía de otros dos espacios o "piezas supletorias" dentro del edificio. A una de ellas se le llamó "salón alto", precisamente por encontrarse encima del salón principal, del que es como una segunda planta superpuesta y simétrica. [A partir de 1593] fue destinado para guardar los libros duplicados y los prohibidos. La segunda de las dos "piezas supletorias" o complementarias del salón principal es el denominado "Salón de verano", que tiene unos 15 metros de largo por 6 de ancho. En su momento fue la sala destinada a los manuscritos y estaba a su vez dividida en dos estancias. En la primera, que era la mayor, se encontraban los manuscritos griegos y latinos; en la segunda, los hebreos, árabes, italianos, castellanos, persas, turcos y otros en lenguas vulgares" (RBME n.d.: "Otros recintos bibliotecarios"). 
pendant le séjour de la cour ${ }^{38}$, et les bibliothécaires ne refusent des livres à personne" (1788: 193). Plantea el caso de dos profesores extranjeros a cuya disposición pusieron los jerónimos los fondos sin ningún tipo de restricciones ${ }^{39}$. Por todo ello, los monjes que atienden la biblioteca le parecen muy gentiles y su labor encomiable.

Si vous aviez apporté à l'Escurial des préjugés contre les Espagnols en général, $\&$ contre les Moines en particulier, vous les déposeriez à coup sûr après avoir passé un quart d'heure avec les Hyéronimites de ce Monastère; vous seriez convaincus que sous le manteau \& même sous le froc Espagnol, se cachent souvent plus de prévenance, plus de complaisance, plus de véritable bonté, que n'en promet l'élégance de nos formes Françoises. J'en appelle à deux Professeurs Danois ${ }^{40}$ qui, amenés il y a quelques années à l'Escurial pour y faire des recherches savantes, furent accueillis par ces Religieux, malgré la différence de mœurs, de langage \& sur-tout de religion, mieux peut-être qu'ils ne l'eussent été à l'université de Coppenhage. On les logea dans le Couvent; on y pourvut à tous leurs besoins, avec toutes les recherches de l'hospitalité la plus généreuse. Tous les trésors de la Bibliotheque leur furent ouverts, $\&$ ils passerent deux mois à feuilleter \& à extraire tous les manuscrits qui tenterent leur curiosité. Ils ont emporté dans leur Patrie des cœurs pénétrés de reconnoissance, $\&$ des porte-feuilles enrichis des fruits de cette laborieuse retraite. La générosité obligeante qu'ils éprouverent en cette occasion est d'autant plus remarquable, que les manuscrits qu'on leur livroit ne sont encore connus du public que par des extraits qu'en a donnés un savant Moine, nommé Cassiri ${ }^{41}$. (1788: 191-192).

Entre los detalles que más sorprenden a Bourgoing está la disposición de los libros en los estantes, "placés à rebours, de manière que leur tranche est tournée en dehors, \& contient leur inscription en longueur" (1788: 193). Al preguntar la razón de esa rareza,

38 La corte es un factor a tener en cuenta ya que aporta animación al espacio puesto que "si la Cour n'y venoit pas porter tous les ans le faste qui l'accompagne, l'Escurial ne seroit plus qu'un vaste couvent” (1788: 197).

39 Antes que ellos, el noble escocés David Colville estuvo trabajando en el establecimiento como catalogador de sus fondos entre 1616 y 1626, aproximadamente, por ser "muy perito en lenguas antiguas, hebreo, griego y árabe" (Andrés 1970:15).

40 Se trata de Thomas Christian Tychsen (1758-1834) y de Daniel Gotthilf Moldenhawer (1753-1823), orientalistas en la Universidad de Göttingen (Gotinga). Probablemente la edición del pastor Plüer del Catalogus Manuscriptorum Bibliothecae Scorialiensis en 1771 debió de influir "en su deseo de visitar la tan famosa biblioteca del Escorial" (Friederich-Stegmann 2014: 137-138). No hay que confundir a T.C. Tychsen, "con el también orientalista Oluf Gerhard Tychsen (1734-1815), igualmente profesor de idiomas orientales en la Universidad de Göttingen, quien mantuvo una disputa epistolar sobre numismática con Francisco Pérez Bayer, bibliotecario en El Escorial.” Él no visitó España. (Friederich-Stegmann 2014: 136-137, nota 597). T.C. Tychsen "había recibido una beca danesa para acompañar, en 1783, al teólogo y bibliotecario alemán Daniel Gotthilf Moldenhawer de Königsberg, en uno de sus viajes por España, cuyo destino principal fue la biblioteca de El Escorial. Mientras que Moldenhawer no llegó a publicar su relato, el estudio de Tychsen Über den gegenwärtigen Zustand der Literatur in Spanien [Sobre el estado actual de las letras en España] se añadió al segundo tomo de la edición alemana del libro de Bourgoing (Friederich-Stegmann 2014: 85-86). Bourgoing no incluye en su obra la identidad de dichos profesores hasta las reediciones posteriores a su nombramiento, en 1799, como miembro de la Academia de Ciencias de Copenhague y, en 1801, como miembro de la Academia de Bellas Artes de Estocolmo.

41 Al igual que Peyron (1783: I, 24-25), este diplomático francés alaba los logros de Miguel de Casiri. 
le explicaron que se debía a Arias Montano cuya biblioteca había sido el núcleo de la Escurialense y que esta, para mantener la uniformidad, había decidido imitarle ${ }^{42}$.

El último viajero francés incluido en el corpus es Pierre-Louis-Auguste de Crusy, marqués de Marcillac (1769-1824), quien realizó, según sus propias palabras, "un séjour assez long" (1805: vj) correspondiente al periodo de 1793-1794. Con posterioridad, plasma su testimonio en Nouveau Voyage en Espagne en el que pretende hacer justicia a este país contrarrestando los juicios de Fleuriot de Langle ${ }^{43}$ acerca de El Escorial con un enfoque positivo a propósito de la biblioteca y matizando algunas reflexiones de Bourgoing. Por consiguiente, aunque de manera sucinta, Marcillac enumera varios de los títulos conservados y describe la decoración. Captan su atención los materiales preciosos utilizados en el mobiliario y la presencia de objetos curiosos como una piedra imantada de siete libras de peso (1805: 46) a la que también se refirieron Vayrac y Peyron:

M. de Langle a oublié de parler de la bibliothèque contenant quatre mille manuscrits hébreux, grecs et arabes, parmi lesquels sont les quatre Evangelistes, écrits en lettres d'or; le Traité de saint Augustin de baptismo parvulorum, écrit de la main de ce saint; les Euvres de sainte Thérèse, originales. -Dix-huit mille volumes sont alignés sur des tablettes de bois des Indes, dont la base est un socle de jaspe d'un pied de haut.

Sur une des cinq tables qu'on voit dans la salle principale de la bibliothèque (deux sont en porphyre) on voit Charlemagne entouré des princes de la maison Palatine. Ce monument contient mille huit cent quarante-huit onces d'argent, quarante-trois d'or, et vingt livres de lapis lazuli. On remarque aussi un Philippe IV à cheval. La statue et le cheval sont d'argent, ainsi que le piédestal qui est orné de lapis lazuli. Les ancêtres de la reine Anne de Neobourg, femme de Charles II, occupent un petit temple en argent, posé sur une des tables en marbre. On voit aussi une pierre d'aimant, du poids de sept livres. Le couvent de l'Escurial est habité par deux cents Hyéronimites. Ils sont fort riches (1805: 46-47).

La relación de este oficial francés proporciona los mismos datos, en cuanto al número de monjes que componen la comunidad religiosa del monasterio, que el texto de Silhouette y que los relatos de Saint-Simon, Silhouette y Beaumarchais respecto a su opulencia (1805: 47), aserto infundado del que, al igual que ellos, tampoco aporta justificación.

Son numerosos los aspectos de la biblioteca de El Escorial en los que se detienen los escritores recogidos en este estudio: la estructura arquitectónica y decorativa de las distintas salas, la organización de los fondos, la formación de las colecciones, las

42 La razón apuntada por Bourgoing no es cierta ya que, por disposición del P. Sigüenza, "se colocaron los libros impresos de autores antiguos, como griegos, latinos y hebreos, en el Salón de los Frescos, con los cantos dorados hacia fuera para resaltar el brillo del oro" (Andrés 1970: 14).

43 Este autor francés incluye en su Voyage de Figaro en Espagne (1784) una entrada titulada "L'Escurial" en la que menoscaba a Felipe II y la obra que mandó construir. 
referencias a los títulos más valiosos o raros, la alusión a figuras clave en la historia de la Laurentina, o la crítica hacia los monjes a su cargo, por ejemplo. Los testimonios de Vayrac, Peyron y Bourgoing son los más pormenorizados porque quizás se inspiran en el tratado de biblioteconomía del jesuita Claude Clément Musei, sive Bibliothecae tam privatae quam publicae Extructio, Instructio, Cura, Usus, Libri IV. Accessit accurata descriptio Regiae Bibliothecae S. Laurentii Escurialis (1635), de enorme difusión durante los siglos XVII y XVIII. Por el contrario, el laconismo caracteriza los de Saint-Simon y Silhouette; Beaumarchais y Marcillac, siendo también parcos, se explayan un poco más. Estas diferencias pueden explicarse, en parte, según el lapso de tiempo que pasan en El Escorial. Otros motivos serían el propio fin del viaje, el pensamiento de cada autor, su peculiar estilo literario o incluso la naturaleza de sus fuentes. Es patente que hay temas que suelen repetirse de un documento a otro, sin embargo no siempre son abordados del mismo modo. Respecto a los datos que aportan, en la mayoría de casos la información brindada es cierta, pero, a veces, no coincide con la realidad. Por ende, la presencia de la Escurialense en las obras analizadas completa, sin duda, el panorama sobre las bibliotecas españolas que ofrecen los viajeros franceses del Siglo de las Luces en sus relatos.

\section{BIBLIOGRAFÍA}

AGUILÁ-SOLANA, Irene (2017): "Las bibliotecas españolas según algunos viajeros franceses del siglo XVIII". Estudios Románicos, 26: 11-22. [https://revistas. um.es/estudiosromanicos/article/view/310931; 09/04/2019]

ÁLVAREZ CABANAS, Andrés, "Un cantoral agustiniano", ABC Madrid 07/09/1930: 11; [http://hemeroteca.abc.es/nav/Navigate.exe/hemeroteca/madrid/abc/1930/09/07/011. html; 16/04/2019]

ANDRÉS, Gregorio de (1970): La Real Biblioteca de El Escorial. Madrid: Aldus.

BEAUMARCHAIS, Pierre de (1858): "Lettre écrite par Beaumarchais au duc de La Vallière, à Madrid, le 24 décembre 1764" dans Louis de Loménie, Beaumarchais et son temps: études sur la société en France au XVIIIe siècle d'après des documents inédits, París: Michel-Lévy frères, 2 vol., t. I: 502-507.

BLASCO CASTIÑEYRA, Selina (1991): "La imagen literaria de El Escorial en el siglo XVIII. Reflexiones sobre las fuentes del viaje ilustrado". Cuadernos de Historia Moderna, 12: 167-182.

BONET BLANCO, M. ${ }^{a}$ Concepción (1996): "La imagen del Escorial: Un viaje a través de la literatura (siglos XVII y XVIII)", Literatura e Imagen en El Escorial. F. J. Campos y Fernández de Sevilla (coord.). Real Centro Universitario Escorial-María Cristina, 511-530.

BOURGOING, Jean-François de (1788): Nouveau Voyage en Espagne, ou Tableau de l'état actuel de cette Monarchie. París: Regnault, 3 t., t. I.

FEDUCHI RUIZ, Luis (1963): "El mueble en las colecciones de El Escorial”, El Escorial 1563-1963. II, Arquitectura, artes. Madrid: Patrimonio Nacional, 521-550. 
FRIEDERICH-STEGMANN, Hiltrud (2014): La imagen de España en los libros de los viajeros alemanes del siglo XVIII. Sant Vicent del Raspeig: Publicaciones de la Universidad de Alicante.

GARCÍA ROMERAL, Carlos (2000): Bio-bibliografía de viajeros por España y Portugal: (siglo XVIII). Madrid: Ollero \& Ramos.

HERNÁNDEZ-DON BUESO, Juan José (1996): “Cronistas extranjeros en El Escorial”, Literatura e Imagen en El Escorial, F. J. Campos y Fernández de Sevilla (coord.). Real Centro Universitario Escorial-María Cristina, 599-628.

JIMÉNEZ DOMINGO, M. ${ }^{a}$ Elena (2006): Las obras gramaticales del abate Jean de Vayrac (1664-1734?) "traductor" de Felipe V [Tesis Doctoral] Valencia. [https://www.uv.es/lepinet/Tesis\%20doctoral\%20M.elena\%20Jimenez.pdf]

LAS PALMAS, Fray Antonio de (1963): "La orden jerónima y El Escorial", El Escorial 1563-1963. I, Historia, literatura. Madrid: Patrimonio Nacional, 399-416.

MARCILLAC, Pierre-Louis-Auguste, marquis de Crusy (1805): Nouveau voyage en Espagne, París: Le Normant.

Manuscritos árabes. [http://rbme.patrimonionacional.es/home/Sobre-la-Biblioteca/ Composicion-del-fondo-bibliografico-manuscrito/Manuscritos-arabes.aspx; 26/04/2019]

Manuscritos castellanos. [http://rbme.patrimonionacional.es/home/Sobre-la-Biblioteca/Composicion-del-fondo-bibliografico-manuscrito/Manuscritos-castellanos.aspx; 26/04/2019]

Manuscritos griegos. [http://rbme.patrimonionacional.es/home/Sobre-la-Biblioteca/ Composicion-del-fondo-bibliografico-manuscrito/Manuscritos-griegos.aspx; 26/04/2019]

Manuscritos hebreos. [http://rbme.patrimonionacional.es/home/Sobre-la-Biblioteca/ Composicion-del-fondo-bibliografico-manuscrito/Manuscritos-hebreos. aspx; 26/04/2019]

Manuscritos latinos. [http://rbme.patrimonionacional.es/home/Sobre-la-Biblioteca/ Composicion-del-fondo-bibliografico-manuscrito/Manuscritos-latinos.aspx; 26/04/2019]

Otros recintos bibliotecarios. [http://rbme.patrimonionacional.es/home/Sobre-la-Biblioteca/Salon-Principal/Otros-recintos-bibliotecarios.aspx; 16/04/2019]

PEYRON, Jean-François (1783): Essais sur l'Espagne. Nouveau Voyage en Espagne fait en 1777 et en 1778. Londres: P. Elmsly, 2 tomos.

Pinturas al fresco. [http://rbme.patrimonionacional.es/home/Sobre-la-Biblioteca/Salon-Principal/Pinturas-al-fresco.aspx; 16/04/2019]

Pinturas al óleo. [http://rbme.patrimonionacional.es/home/Sobre-la-Biblioteca/SalonPrincipal/Pinturas-al-oleo.aspx; $; 16 / 04 / 2019]$

El recinto. [http://rbme.patrimonionacional.es/home/Sobre-la-Biblioteca/Salon-Principal/El-recinto.aspx; 16/04/2019]

RBME: Real Biblioteca del Monasterio de San Lorenzo de El Escorial (n.d.). Composición del fondo bibliográfico impreso. [http://rbme.patrimonionacional.es/ 
home/Sobre-la-Biblioteca/Composicion-del-fondo-bibliografico-impreso. aspx; 26/04/2019]

SAINT-SIMON, Louis de Rouvroy, duc de (1858): Mémoires complets et authentiques du duc de Saint-Simon sur le siècle de Louis XIV et la Régence, París: Hachette, t. XVIII.

SILHOUETTE, Étienne de (M. S***) (1770) : Voyages de France, d'Espagne, de Portugal et d'Italie. París: Merlin, t. IV.

SCHOLZ-HANSEL, Michael (1992-1993): "Las obras de Pellegrino Tibaldi en el Escorial: un resumen original del Arte italiano de su tiempo". Imafronte, $\mathrm{n}^{\circ} 8-9$ : $389-401$.

VAYRAC, abbé de (1718): État présent de l'Espagne où l'on voit une Géographie Historique du Pays, etc., París: André Cailleau, 2 tomos, t. I.

WEISS, Charles (1841): Biographie universelle ou Dictionnaire historique, París : Furne et $C^{\text {ie }}$. t. VI.

\section{PERFIL ACADÉMICO Y PROFESIONAL}

Irene Aguilá-Solana es doctora en Filología Francesa y profesora titular de Filología Francesa en la Universidad de Zaragoza. Su tesis versó sobre los temas y las formas dramáticas en el "Théâtre de la Foire" del siglo XVIII. Todas sus líneas de investigación convergen en la literatura francesa del Siglo de las Luces y abarcan los estudios de recepción, los relatos de viajes, así como la escritura libertina, la cuentística y el género epistolar.

Fecha de recepción: 13/05/2019

Fecha de aceptación: 02/06/2019 